\title{
FIB Preparation and STEM Studies of Earthworm Spherulite Crystallization
}

\author{
J. Méndez*, J. B. Rodríguez-González*, L. Gago-Duport** and M.J.I. Briones*** \\ * Servicio de Microscopía Electrónica, CACTI. Universidad de Vigo, 36310 Vigo, Spain \\ ** Dept. Geociencias Marinas, Universidad de Vigo, 36310 Vigo, Spain \\ *** Dept. de Ecología y Biología Animal, Universidad de Vigo, 36310 Vigo, Spain
}

The species of earthworms included in the Lumbricidae family have a very complex esophageal organ called calciferous or Morren gland, being the representatives of the genus Lumbricus the individuals where that gland is better developed. Previous studies $[1,2]$ have shown the secretion produced in the glandular structure is not crystalline but amorphous in the form of amorphous calcium carbonate (ACC) spherulites. Afterwards the spherulites undergo a crystallization process to form rhombohedral calcite crystals that finally result in macroaggregates of these crystals. This crystallization process can go through two intermediate states, vaterite or aragonite. These changes happen on the surface of the amorphous phases.

To achieve a detailed knowledge over the growth and crystallization process of this secretion, we have studied the internal structure of the spherulites using the focused ion beam (FIB) technique to prepare cross-section lamellae of the centre of the spherulites (Figure 1). To do that, the first step is the smashing of the glandular segments over a glass slide surface to take out the leachate.

In Figure 2 we show two cross-section images of the lamellas obtained in the scanning electron microscope with the dark field (DF) transmission detector (SEM-STEM). From these images we conclude that there are at least two different types of spherulites. The first type has several domains well crystallized (Figure 2A), while the second type has a clear concentric structure where crystalline domains aren't revealed (Figure 2B). It is not clear at this point whether these represent two different stages of crystallization or two different forms of spherulite crystallization.

\section{References}

1. Gago-Duport L. et al., J. Struct. Biol. 162: 422, 2008

2. Méndez J. et al., EMC 2008, Vol. 3: 127, 2008 


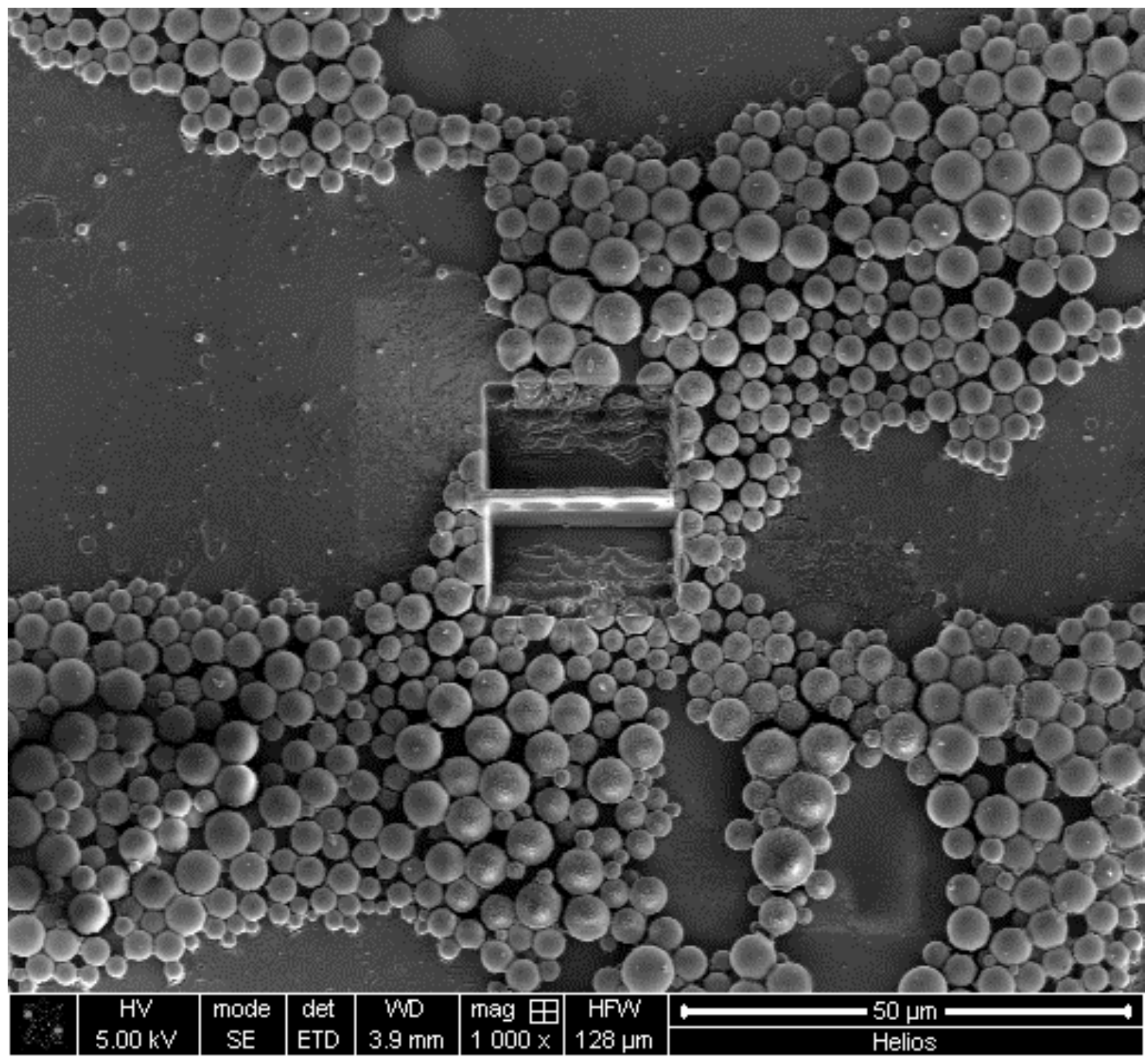

Figure 1. SEM image of dry leached including a large number of spherulites. The image also shows the position where a lamella with three particles was extracted.
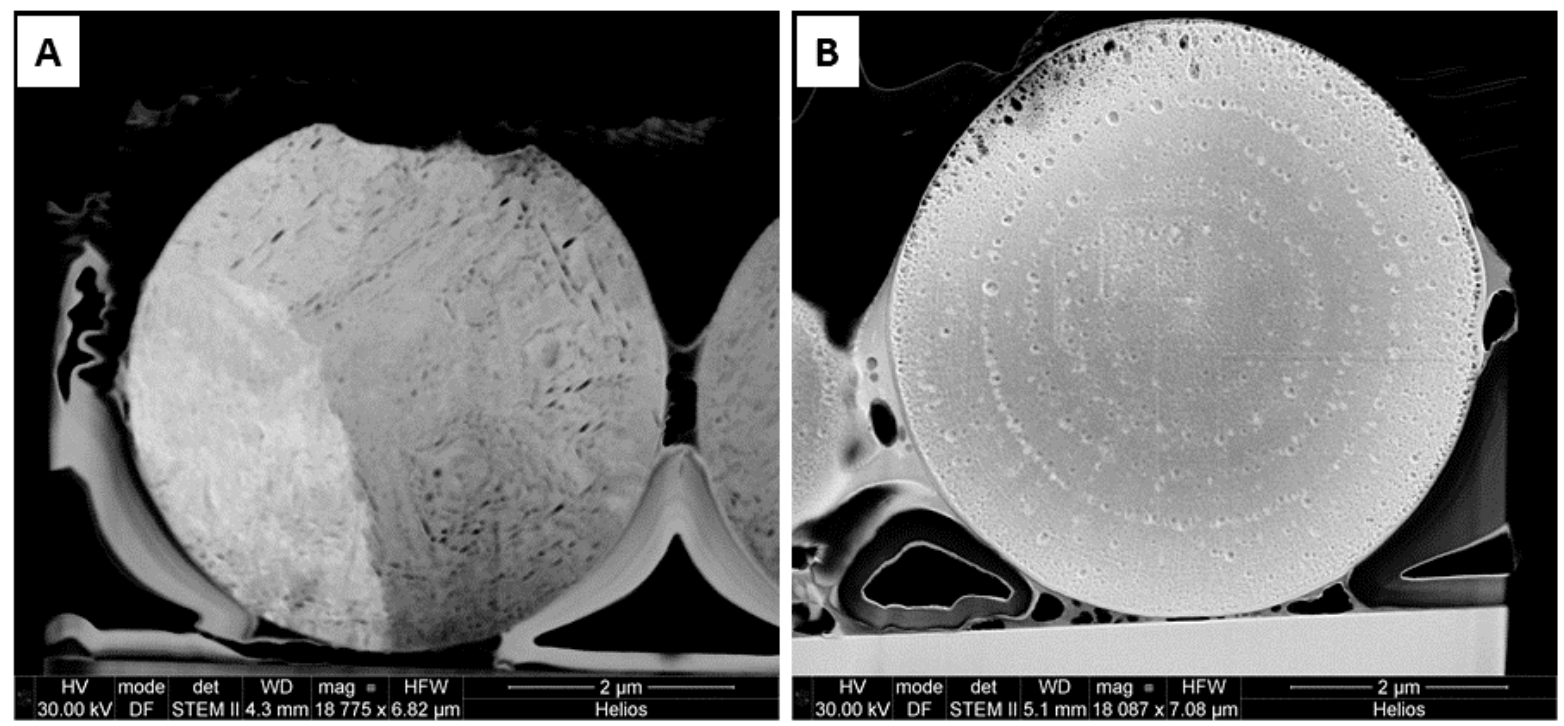

Figure 2. Dark field SEM-STEM cross-sections of two different spherulites prepared by FIB. (A) Spherulite showing a polycrystalline internal structure. (B) Spherulite with a concentric structure. 